MOTARJEMI, Y.; VANSCHOTHORST, M.; KÄFERSTEIN, F. Future challenges in global harmonization of safety legislation. Food Control, St Louis, v. 12, p.339-346, 2001.

\title{
FUTUROS DESAFIOS NA HARMONIZAÇÃO DA LEGISLAÇÃO GLOBAL DE SEGURANÇA DOS ALIMENTOS
}

\author{
Por Cyleni Regattieri Abreu de Souza \\ Escola SENAI Horácio Augusto da Silveira, E-mail: cyleni@usp.br
}

A preocupação com as doenças transmitidas por alimentos acompanha o homem desde muito tempo. As doenças transmitidas por alimentos continuam sendo um grande problema para os governos, produtores, prestadores de serviços e consumidores. Segundo a Food Agricultural Organization - FAO (1998), cerca de um terço da população de países desenvolvidos é afetado por essas doenças todos os anos. Nos países em desenvolvimento, a situação é ainda mais séria. Isso significa que as doenças transmitidas por alimentos causam sofrimento humano e perdas econômicas, contribuindo negativamente com a saúde e o bem estar da humanidade e facilitando a disseminação da miséria.

A manifestação desses perigos, assim como a magnitude do efeito que causam, é dependente das condições de obtenção e de produção de alimentos. O Acordo de Aplicações de Medidas Sanitárias e Fitossanitárias (SFS) estabelece que os países podem aplicar medidas sanitárias diferentes, desde que seus resultados finais sejam equivalentes e desde que haja transparência na adoção e na aplicação dessas medidas. Essas medidas devem ser baseadas na avaliação de riscos.

A avaliação de risco é o processo de estimar a probabilidade de perigo causar efeitos adversos numa dada população, sob condições particulares de exposição. Ela fornece uma base racional para as decisões de saúde pública, permitindo ao administrador de saúde pública ponderar sobre os riscos do perigo para saúde humana e os custos advindos das medidas necessárias para redução dos mesmos.

A análise de risco trata dos riscos decorrentes da presença de perigos em alimentos identificando e priorizando esses perigos, levantando-se o que se sabe sobre o perigo, relacionando as organizações públicas e privadas que têm interesse no gerenciamento e na comunicação do risco do perigo.

Esse trabalho tem como objetivo fazer uma análise crítica do artigo que aborda a análise de risco como o caminho para harmonização da legislação de segurança dos alimentos num nível global. A primeira parte da resenha compreende a apresentação das idéias que sustentam o pensamento do autor. Na apreciação crítica, procurou-se avaliar a validade ou aplicabilidade do que foi exposto pelo autor e finalmente nas considerações finais, são apresentadas as principais reflexões e constatações decorrentes do desenvolvimento do trabalho. 
A globalização influencia diferentes aspectos da vida humana, incluindo a segurança na cadeia produtiva de alimentos e a saúde do consumidor. Problemas de segurança dos alimentos de um país podem facilmente se tornar problemas para outros países. 0 comércio internacional de alimentos, viagens internacionais ou migração e mudanças de estilo de vida pela globalização da informação, cultura e valores são fatores que contribuem para os problemas globalizados de segurança dos alimentos.

Todos têm direito a um alimento nutricionalmente adequado e seguro. A harmonização global das legislações sobre segurança dos alimentos garante a competição justa entre os países em termos de comércio, ao mesmo tempo em que garante que toda população mundial tenha o mesmo grau de segurança de alimentos.

Os acordos de SFS e de BTC foram particularmente importantes para a segurança dos alimentos e tiveram um grande impacto na harmonização da legislação pertinente.

A Organização Mundial do Comércio (OMC), criada em 1994 em decorrência do antigo General Agrrement on Tariff an Trade (GATT), reviu os acordos SFS, proposto pela Rodada do Uruguai e o BTC proposto pela Rodada de Tóquio. Ambos ordenam uma mesma linha de atuação para pareceres internacionais em caso de divergência e disputa entre os países. Essa linha de atuação deve ser baseada em padrões internacionais, guias e recomendações existentes como as da Comissão Codex Alimentarius (CAC).

Os pareceres podem aceitar outras medidas sanitárias e fitossanitárias equivalentes se o país exportador demonstrar ao país importador que seus padrões garantem o nível apropriado de proteção à saúde. O questionamento do que é nível apropriado de proteção à saúde humana foi levado aos fóruns internacionais. As reuniões da FAO e Organização Mundial da Saúde (OMS) no final dos anos 90 introduziram o conceito de análise de risco. Neste contexto, a base para as decisões relacionadas à segurança dos alimentos deve adotar princípios racionais e lógicos baseados em dados científicos e considerações culturais e socioeconômicas.

Segundo os autores, não é possível discutir harmonização de legislação em segurança dos alimentos sem relacionar a avaliação e a comunicação do risco. A legislação de segurança dos alimentos é uma opção do gerenciamento do risco e se baseia no levantamento de dados através da avaliação de risco e na comunicação do risco.

No artigo ainda são levantados os desafios científicos e sociais na harmonização da legislação de segurança de alimentos. Os desafios científicos referem-se a todas as atividades e esforços necessários para levantar dados científicos para subsidiar decisões apropriadas e transparentes. A chave para harmonização da legislação sobre alimentos seguros é alcançar acordos que forneçam proteção adequada ao consumidor, inclusive ao bem estar animal e às questões do meio ambiente, que não comprometam os benefícios do crescimento econômico e do progresso da ciência e tecnologia, e que não penalizem os países em desenvolvimento e as pequenas empresas, bem como as sociedades mais fracas. Algumas medidas como aumentar a participação do setor público nos trabalhos da CAC, aumentar o diálogo e a comunicação com o consumidor e promover ações 
de educação em segurança dos alimentos podem contribuir para a harmonização da legislação em segurança dos alimentos. Além disso, deve-se disseminar informações, experiências e conhecimento gerados na universidade, na indústria, no setor público e nas organizações não governamentais para se concentrar esforços para promover a segurança dos alimentos globalmente.

Alguns fatores citados pelos autores realmente contribuem para os problemas globalizados de segurança dos alimentos. O surgimento de microrganismos emergentes está diretamente relacionado com a facilidade de circulação mundial de pessoas e produtos. Um microrganismo patogênico transferido de um país a outro através desta via pode produzir efeitos de grande impacto sanitário, social e econômico sobre uma população que não está acostumada à exposição a este perigo específico ( $\mathrm{SCHUCH}$, 2008).

Um exemplo de mudanças de estilo de vida pela globalização da informação, cultura e valores é a introdução da culinária de outros países no cotidiano das pessoas. Em 2004, a investigação do Centro de Vigilância Epidemiológica do Estado de São Paulo (CVE) concluiu que o salmão consumido cru em pratos típicos da cozinha japonesa foi responsável pelo surto de difilobotríase, infecção intestinal causada pela "tênia do peixe" (COLLUCCI, 2005).

No acordo BTC há o reconhecimento do direito dos países adotarem normas que considerem apropriadas, por exemplo, para a proteção da saúde e da vida de pessoas e dos animais ou a preservação dos vegetais, para a proteção do meio ambiente ou em defesa de outros interesses dos consumidores. Estes são, segundo o acordo, objetivos legítimos para a adoção de medidas restritivas. O acordo BTC estabelece propósito legítimo dos padrões e regulamentos técnicos; custo da implementação proporcional à finalidade do padrão; uso de métodos menos restritivos ao comércio e uso, pelos países-membros, de padrões e regulamentos internacionais no âmbito de seu território; não tolerância de exigências tecnológicas e de especificações de produtos que não são aplicadas internamente ou não são alcançáveis pelo país importador (RASZL, 2007).

A eliminação de barreiras é o objetivo principal. Entretanto, algumas medidas são adotadas por parte dos países desenvolvidos com vistas à proteção de seus mercados, a despeito da existência desses acordos. Na maioria das vezes, essas "barreiras" ao comércio se devem à utilização de normas ou regulamentos técnicos não-transparentes ou não-embasados em normas internacionalmente aceitas ou, ainda, decorrentes da adoção de procedimentos de avaliação da conformidade não-transparentes e/ou demasiadamente dispendiosos, bem como de inspeções excessivamente rigorosas. Trata-se das chamadas "Barreiras Técnicas ao Comércio" (FERMAM, 2003). Por exemplo, a projeção do European Coffee Federation de estabelecer um limite máximo de 5 ppb de ocratoxina A (OTA) no café verde. Isso poderia envolver um aumento nas taxas de rejeição do café exportado de $7 \%$ a $18 \%$, o que preocupa países produtores de café. Esse limite para OTA estabelecido pela União Européia pode causar impacto econômico em países produtores desses grãos, como o Brasil (GOLLÜCKE; TANIWAKI; TAVARES, 2004). 
Os Acordos SFS e BTC, citados pelos autores, elegeram as diretrizes e recomendações do Codex Alimentarius como sendo medidas que devam ser preferencialmente adotadas pelos países com vistas a facilitar o comércio de alimentos. O Codex Alimentarius é um ponto de referência mundial de grande relevância para consumidores, produtores, fabricantes, organismos nacionais de controle e o comércio internacional de alimentos.

No Codex, os países participam na harmonização e aplicação de normas relativas a alimentos em escala mundial, além da possibilidade de participarem na elaboração de normas alimentares de uso internacional. Há o reconhecimento de que as normas do Codex possuem base científica sólida, contudo, deve-se levar em consideração os seguintes elementos: a medida sanitária deve ser embasada nas evidências científicas disponíveis, deve-se considerar os métodos de produção, as formas de inspeção,os métodos de coleta de amostras, a garantia da qualidade analítica, a prevalência de doenças ou pestes específicas, as condições relevantes do meio ambiente e da ecologia, a quarentena e outros tratamentos e processos relevantes. Quando as evidências científicas são insuficientes, os países podem adotar medidas sanitárias provisórias, com base em informações pertinentes. Porém, em um espaço razoável de tempo, para geração de evidências científicas, essas medidas provisórias devem ser revistas (RASZL, 2007).

A FAO e a OMS organizaram conferências no final dos anos 90 onde o conceito de análise de risco foi introduzido para embasar as decisões relativas à segurança dos alimentos. $O$ artigo $5^{\circ}$ do Acordo SPS estabelece a importância do desenvolvimento da avaliação de risco microbiológico (MRA) pelos países membros, o que visa à disponibilização de informação cientificamente obtida para dar suporte à elaboração de padrões, guias e recomendações relacionadas com a inocuidade dos alimentos ( $\mathrm{SCHUCH}, 2008)$. O processo de análise de risco é constituído de três componentes: avaliação, gestão e comunicação de riscos. A metodologia trata dos riscos decorrentes da presença de perigos em alimentos identificando e priorizando esses perigos, levantando-se o que se sabe sobre o perigo, relacionando as organizações públicas e privadas que têm interesse no gerenciamento e na comunicação do risco do perigo (RASZL, 2007).

A avaliação de risco é o processo de estimar a probabilidade de um agente químico ou biológico causar efeitos adversos numa dada população, sob condições particulares de exposição. Ela fornece uma base racional para as decisões de saúde pública, permitindo ao administrador de saúde pública ponderar sobre os riscos do perigo para saúde humana e os custos advindos das medidas necessárias para redução dos mesmos (PAUMGARTTEN, 1993).

O autor ressalta a complexidade da avaliação do risco dos perigos biológicos em comparação aos perigos químicos devido a variações consideráveis entre os países, principalmente entre os países desenvolvidos e em desenvolvimento. O que é procedente, pois a avaliação de risco é fundamentalmente um exercício científico, e para a tomada de decisão, a confiabilidade depende, em grande parte, da adequação da base científica utilizada. Quanto mais adequada e mais completa for essa base de dados, menor será o grau de incertezas na estimativa do risco feita pelo gestor. 
A estimativa do risco deve ser acompanhada não somente por um sumário de qualquer informação biológica relevante disponível, mas também por uma lista de suposições utilizadas com suas limitações, e a evolução crítica das incertezas, qualitativa e quantitativa, na avaliação do risco. Em geral a forma de se compensar as incertezas no processo de avaliação do risco é utilizar fatores arbitrários de segurança quando limites quantitativos para humanos e exposição ambiental serão definidos. Ou seja, é feita uma permissão a fim de se permanecer do lado seguro, mesmo no caso de erro. Segundo Paumgartten (1993), países em desenvolvimento, como os da América do Sul, a confiabilidade e a exatidão devem ser consideradas como prioridades na avaliação do risco.

O processo de avaliação de risco consiste de quatro elementos (NATIONAL ACADEMY OF SCIENCES, 1983): identificação do perigo, avaliação da exposição, avaliação da doseresposta e caracterização do risco.

A identificação do risco é o primeiro estágio e consiste na avaliação da natureza e da força das evidências da relação causa e efeito em estudos laboratoriais com animais ou seres humanos. A identificação do perigo poderá fornecer respostas para questão: A exposição ao perigo causa efeitos adversos à saúde?

A avaliação da exposição consiste na estimativa da quantidade do perigo ingerida ou absorvida por humanos submetidos à exposição do perigo por diferentes rotas. $O$ tempo de exposição e o número de indivíduos expostos, ou ainda a serem expostos, são parâmetros que também precisam ser levados em conta. A avaliação da exposição poderá responder a questão: Que níveis da exposição estão sendo experimentados atualmente por indivíduos, ou pode ser antecipado para aqueles indivíduos, sob circunstâncias diferentes? Ou seja, na avaliação do risco de um perigo em particular deve levar em consideração circunstâncias da região, da população, do grau desenvolvimento, dentre outros.

A relação dose-resposta preocupa-se com a evolução da relação entre a quantidade do agente e a expectativa de incidência do efeito adverso na população. Durante esta fase da avaliação do risco a pergunta a ser respondida é: Qual a relação entre a quantidade do agente e a incidência nas reações adversas em humanos?

A caracterização do risco é baseada nas três etapas anteriores e constitui a saída da avaliação do risco como um todo. Os dados levantados e analisados nos três estágios anteriores são apresentados, integrados e resumidos e as conclusões sobre o risco de efeitos adversos à saúde são extraídas. Assim, na caracterização do risco, a informação é disponibilizada de forma útil e facilmente entendida pelos gestores de risco que são responsáveis por tomarem decisões nos problemas relacionados à saúde pública. Nesta etapa, a pergunta a ser respondida é: Qual é a estimativa da incidência de efeitos adversos à saúde numa dada população, sob condições específicas de exposição? Entretanto, como enfatizaram Preuss \& Ehrlich (1987) e Paustenbach (1989), a resposta a essa questão deve sempre ser qualificada. Ou seja, ela deve vir acompanhada de um resumo baseado em informações biológicas relevantes, as premissas utilizadas e as limitações dessas premissas, junto com uma análise crítica de qualquer incerteza qualitativa e quantitativa. 
O gerenciamento do risco é responsável em priorizar e caracterizar o que se sabe sobre o mesmo e que intervenções são possíveis para controlar os riscos, dimensionar as alternativas, avaliar a aplicação das mesmas através de consulta a todas as partes interessadas e envolvidas, com o objetivo de promover a segurança dos alimentos e, quando necessário, selecionar as opções usadas para a prevenção e o controle apropriado do perigo e respectivo controle que poderá ou deverá ser aplicado em um, dois ou todos os elos da cadeia produtiva de alimentos (RASZL, 2007).

A comunicação do risco é uma troca interativa de informações e opiniões a respeito do risco entre os avaliadores e gestores de risco, consumidores e outras partes interessadas para promover a divulgação e compreensão do tema específico sob consideração durante o processo de análise de risco (RASZL, 2007).

O uso do processo de análise de riscos em âmbito internacional tem demonstrado o seu valor e capacidade para melhoria contínua de controle de perigos e riscos, além de facilitar o processo de tomada de decisões. Diversas atividades conjuntas de apoio ao CAC têm sido levadas a cabo pela OMS e FAO para o tratamento dos temas prioritários apontados pelos países, através da identificação e formação de grupos de trabalhos de expertos em cada uma das áreas específicas. Em julho de 2000, especialistas se reuniram em Roma para tratamento dos seguintes assuntos: caracterização do perigo Salmonella em aves e ovos; avaliação de exposição para Salmonella Enteritidis em ovos e em aves; identificação e caracterização de perigo Listeria monocytogenes em alimentos prontos para o consumo (SCHUCH, 2008).

Os autores reforçam no artigo a necessidade de se levar em consideração os desafios científicos e sociais decorrentes da aplicação da análise de risco para a harmonização da legislação em segurança de alimentos num nível global.

Os desafios científicos referem-se, neste contexto, a todas atividades e esforços necessários para levantar dados científicos que embasem decisões transparentes e apropriadas. O acesso à segurança dos alimentos com base na ciência não é uma atividade completamente nova: está relacionado a processos como os de Boas Práticas Agrícolas, Boas Práticas de Higiene, Boas Práticas de Fabricação e com o Sistema de Análise de Perigos e Pontos Críticos de Controle (APPCC), entre outros. O que é novo é o uso da análise de riscos como uma viga mestra para detectar e responder aos problemas de segurança por intermédio de uma forma sistemática, estruturada e científica, com o objetivo de acessar a qualidade da tomada de decisões ao longo da cadeia de produção de alimentos (RASZL, 2007). A aplicação pura e simples da metodologia de análise de risco não garante a segurança dos alimentos. É necessário aprimoramento e investimentos na infra-estrutura da cadeia produtiva dos alimentos e do sistema de saúde.

Os desafios sociais referem-se ao equilíbrio entre os interesses dos países desenvolvidos, ou industrializados, e dos países em desenvolvimento, que pode ser superado através de programas de cooperação internacional, ao equilíbrio entre o desenvolvimento econômico e a segurança dos alimentos, que não deve comprometer o fornecimento de alimentos nutricionalmente adequados e seguros à população. 
A avaliação de risco tem sido usada em nível internacional para desenvolver medidas sanitárias e fitossanitárias. A FAO, a OMS e Codex Alimentarius tem trabalhado intensamente para que os padrões internacionais tenham por base a ciência. A avaliação de risco em nível internacional espelha os riscos dos países em que está sendo desenvolvida. Esse é um problema internacional e em especial para os países que ainda não iniciaram as atividades de avaliação de riscos, pois esses países, além de não fornecerem seus dados, têm que aceitar as decisões tomadas com base nos dados existentes. Ainda, o cenário que pode ser estabelecido para um agente-alimento a ser comercializado em nível internacional pode não ter nenhuma base ou evidência científica e ser desfavorável para um dos países envolvidos.

A decisão das formas de gestão pelos produtores deve ter por base uma avaliação de riscos para a construção de parcerias que visam à segurança do produto final, para avaliar e validar as formas de controle e para dar garantia de que os níveis do perigo no produto final são os esperados. Ainda, em questões legais e de reclamações, o setor produtivo tem base para caracterizar fatos e evidências científicas que permitem tomadas de decisão sobre todos os aspectos de controle, como a identificação dos perigos significativos ou prováveis e a sua relação com os pontos críticos de controle (PCC) e os procedimentos operacionais, assim como as estratégias de controle ou medidas adotadas para o controle dos mesmos (limites críticos, formas de monitorização, ações corretivas e programas de verificação).

A análise de risco não é operacional, porém sem ela a operacionalização não demonstra a transparência e equivalência dos resultados de controle necessários para qualificação de programas de Boas Práticas e do Sistema APPCC.

O processo de análise de riscos é formulado como base e suporte para a tomada de decisão para as intervenções operacionais de controle de perigos, porém enfocando especificamente doenças e priorizando os perigos para fins de controle, considerando a magnitude do efeito adverso que pode desencadear no consumidor. Esse processo deve ser flexível para atender as múltiplas necessidades associadas à segurança dos alimentos.

O processo de análise de riscos tem por foco as doenças e agravos à saúde pública do consumidor, fornece base científica aos objetivos e às decisões para o controle operacional de perigos e respectivos riscos, permite a conjugação estreita e interdependente da ciência, dos dados científicos e das atividades de gerenciamento de riscos, é aplicável pelo setor governamental e também pelo setor produtivo, promove o desenvolvimento e refinamento indireto de dados e estudo sobre doenças transmissíveis por alimentos, promove o estudo e a reflexão do desenvolvimento científico e estabelece regras gerais e parcerias necessárias para a tomada de decisão de controle de perigos em alimentos e para a verificação de sua efetividade. 


\section{REFERÊNCIAS}

COLLUCCI, C. Salmão causou surto de doença, diz laudo. Folha de São Paulo, São Paulo, 02 junho 2005. Disponível em:<http://www1.folha.uol.com.br/folha/ cotidiano/ult95u109627.shtml>. Acesso em: 24 outubro 2008.

EUROPEAN COMMUNITY. Commission Regulation (EC) No 472/2002 of 12 March 2002 amending Regulation (EC) No 466/2001 setting maximum levels for certain contaminants in foodstuffs. Official Journal of the European Communities, [S.I.], L75, v. 45, p. 18-20, 2002. Disponível em: < http://eur-lex. europa.eu/JOIndex.do?year $=2002 \&$ serie $=$ L\&textfield2 $=75 \&$ Submit $=$ Search \& submit=Search\&ihmlang=en>. Acesso em: 29 fevereiro 2009.

The apllication of risk communication to food standards and safety matters. Report of Joint FAO/WHO Expert Consulation, Rome, 2-6 February, 1998, FAO Food and Nutrition Paper, No. 70, Food and Agriculture Organization of United Nations, 1998

FERMAM, R. K. S. HACCP e as barreiras técnicas. Ponto Focal de Barreiras Técnicas às Exportações Portal. Disponível em: <http://www.inmetro.gov.br/ barreirastecnicas>. Acesso em: 23 out. 2008.

GOLLUCKE, A. P. B.; TANIWAKI, M. H.; TAVARES, D. Q. Survey on ochratoxin A in Brazilian green coffee destined for exports. Ciência e Tecnologia de Alimentos, Campinas, v. 24, n. 4, Dec. 2004 . Disponível em: <http://www.scielo.br/scielo. php?script=sci_arttext\&pid=S0101-20612004000400027\&lng=en\&nrm=iso >. Acesso em: 27 Feb. 2009.

NATIONAL ACADEMY OF SCIENCES. Risk assessment in Federal Government: managing the process. Washington, DC: National Academy Press, 1983. 202 p.

PAUMGARTTEN, Francisco J. R.. Risk assessment for chemical substances: the link between toxicology and public health. Cadernos de Saúde Pública, Rio de Janeiro, v. 9, n. 4, Dec. 1993. Disponível em: <http://www.scielo.br/scielo. php?script=sci_arttext\&pid=S0102-311X1993000400004\&Ing=en\&nrm=iso >. Acesso em: 27 fev. 2009.

PAUSTENBACH, D. J. Important recent advances in the practice of health risk assessment: Implications for the 1990`s. Regulatory Toxicology and Pharmacology, St Louis, v.10, n.3, p. 204-243, 1989. Disponível em: <http:// www.sciencedirect.com/science?_ob=ArticleURL\&_udi=B6WPT-4DDP52N-HK\&_ user $=10 \&$ \&doc $=1 \&$ fmt $=\&$ _orig $=$ search \&_sort $=d \& v i e w=c \&$ acct $=C 000050221 \&$ _ version $=1 \&$ _urlVersion $=0 \&$ \&userid $=10 \& \mathrm{md} 5=\mathrm{d} 7 \mathrm{afa} 70 \mathrm{eb} 40 \mathrm{~d} 927366 \mathrm{cb} 04 \mathrm{c03212 \textrm {a } 1}$ $6 \mathrm{e}>$. Acesso em: 28 fev. 2009. 
SCHUCH, D. M. T. Segurança dos alimentos: inquietudes e expectativas mundiais. Disponível em: <http://www.fortdodge.com.br/fd/aves/banner-revista/ Revista\%20Aves\%206/novorumo.html>. Acesso em: 23 out. 2008.

RASZL, Simone Moraes et al. Sistema de qualidade em segurança de alimentos ABNT NBR ISO 22000:2006, EUREPGAP e BRC. Florianópolis: SENAI/SC Florianópolis, 2007.

\section{FUTURE CHALLENGES IN GLOBAL HARMONIZATION OF FOOD SAFETY LEGISLATION}

\section{SOBRE A AUTORA}

Graduação em Engenharia Química pela Universidade Federal de Minas Gerais
(1990), Mestrado em Ciência de Alimentos pela Universidade Federal de Minas
Gerais (1993) e Doutorado em Tecnologia Bioquímica-Farmacêutica pela
Universidade de São Paulo (2001).
Atualmente é técnica de ensino da Escola SENAI Horácio Augusto da Silveira,
onde ministra aulas no Cuso Técnico em Processamento de Alimentos e atua na
área de Segurança dos Alimentos implantando e auditando programas de Boas
Práticas de Fabricação - BPF e sistemas de Análise de Perigos e Pontos Críticos
de Controle - APPCC em indústrias de alimentos. É consultora, multiplicadora e
auditora do Programa Alimentos Seguros - PAS.
E-mail: cyleni@usp.br

